\title{
Blister Aneurysm of Middle Cerebral Artery Division: Stent-Assisted Coiling Using Shelfing Technique
}

\author{
Shrikant Londhe ${ }^{1} \quad$ Vipul Gupta ${ }^{1} \quad$ Rajsrnivias Parthasarathy ${ }^{1} \quad$ Hilal Ahmad Ganie ${ }^{1} \quad$ Nishchint Jain ${ }^{1}$
}

${ }^{1}$ Department of Interventional Neuroradiology, Artemis Hospital

Address for correspondence Vipul Gupta, MD, Department of Gurugram, Harayana, India \begin{abstract}
Interventional Neuroradiology, Artemis hospital Gurugram122001, Harayana, India (e-mail: vipulgupta25@gmail.com).
\end{abstract}

\begin{abstract}
Keywords

- blood blister-like aneurysm

- middle cerebral artery

- Leo Plus Baby stent
\end{abstract}

Blister aneurysms pose significant diagnostic and therapeutic challenge to neurointerventionists as well as neurosurgeons. Fragile nature of these aneurysms with involvement of the adjacent parent artery makes surgical options more difficult and complicated. Various endovascular treatment options such as overlapping stent, parent artery occlusion, and flow diverter placement are available in the present era. Though side wall aneurysms can be managed with flow diverter placement, bifurcation aneurysms are difficult to treat with these devices due to possibility of compromised flow in covered branch arteries. Blister aneurysms of the middle cerebral artery (MCA) are rare, and their treatment can be challenging when they are treated by endovascular methods with either overlapping stent or flow diverter placement. The authors report a case of ruptured MCA trifurcation blister aneurysm treated with shelfing technique using braided stent monotherapy along with coil embolization. The patient had good clinical outcome (modified Rankin's scale 1 [mRS-1]) at discharge and complete aneurysm occlusion on follow-up angiogram after 6 months.

\section{Introduction}

Blister aneurysms were described in 1988 by Takashi, as focal small arterial wall defect and subsequent vessel wall bulge covered with thin fibrous tissue at nonbranching site. ${ }^{1,2}$ They differ from the typical saccular aneurysm in terms of histology, morphology, and location. ${ }^{3}$ Among the location, supraclinoid internal carotid artery (ICA) is the most commonly described location, particularly involving the dorsomedial wall. ${ }^{1}$ Other locations such as anterior communicating artery (ACOM) and basilar artery have been described. ${ }^{4}$ Middle cerebral artery (MCA) is one of the rare sites of involvement. Management options for these aneurysms are surgical (wrapping, clipping, trapping, and, if required, extracranial-intracranial bypass) or endovascular (parent artery occlusion, single/overlapping stents along with coiling and flow diverter placement). ${ }^{5-7}$ Certain anatomical locations of blister aneurysm make these treatment options challenging with increased morbidity. MCA bifurcation/trifurcation blister aneurysm is one such location where aforementioned options are often difficult. Surgical treatment has increased risk of both morbidity as well as mortality. We report a case of MCA bifurcation blister aneurysm treated with single (monotherapy) braided stent-assisted coil embolization using shelfing technique that enabled us to achieve good outcome with preserved flow in MCA divisions. To our knowledge, this is the first reported case of MCA divisions blister aneurysm treated with braided stent-assisted coil embolization. We discussed the technical details of the procedure in treating these extremely fragile aneurysms.

\section{Case Report}

\section{Clinical History}

A 64-year-old female patient was referred to our hospital with history of sudden-onset severe headache and vomiting, followed by loss of consciousness. On neurologic examination, she had neck rigidity with altered sensorium (Glasgow coma
License terms

(우 (1) $\ominus \circledast$ 
scale [GCS] of E4V1M6 and WFNS grade IV). The patient had vasospasm-related acute cortical infarcts in left MCA territory resulting in aphasia. No motor deficit was noted. She was evaluated with computed tomography (CT) of the brain, which showed acute subarachnoid hemorrhage (modified Fischer's grade of 3). Diagnostic cerebral angiogram showed focal fusiform dilatation near the origin of superior division of the MCA with small bleb-like blister aneurysm arising from

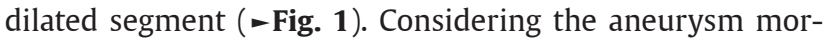
phologic features such as small size, ill-defined wall, stasis of the contrast on delayed angiogram phase, and fusiform dilatation of the base, this was diagnosed to be probable blister aneurysm. Superior division of the MCA measured $1.4 \mathrm{~mm}$ in largest caliber. This case was discussed with the neurosurgery team for possible surgical options. Owing to small size of aneurysm and high risk of intraoperative rupture during the surgery, decision of endovascular treatment was taken.

\section{Technical Considerations}

Preprocedural loading dose of aspirin (300 mg) and ticagrelor (180 mg) was given 2 hours before the procedure. Under GA, 6 F long sheath $(70 \mathrm{~cm}$, Flexor Raabe Sheath, Cook Medical) was placed into the left common carotid artery. After this, $6 \mathrm{~F}$ (0.070 ID) DAC (distal access catheter; Concentric Medical) guiding catheter was placed till the petrous ICA. Three-dimensional (3D) rotational angiography and directed two-dimensional (2D) working angles were taken for the further procedure. Using the Synchro microwire (Stryker Neurovascular) and Vasco-10 microcatheter (Balt Extrusion), superior division of the left MCA was catheterized for the stent placement.
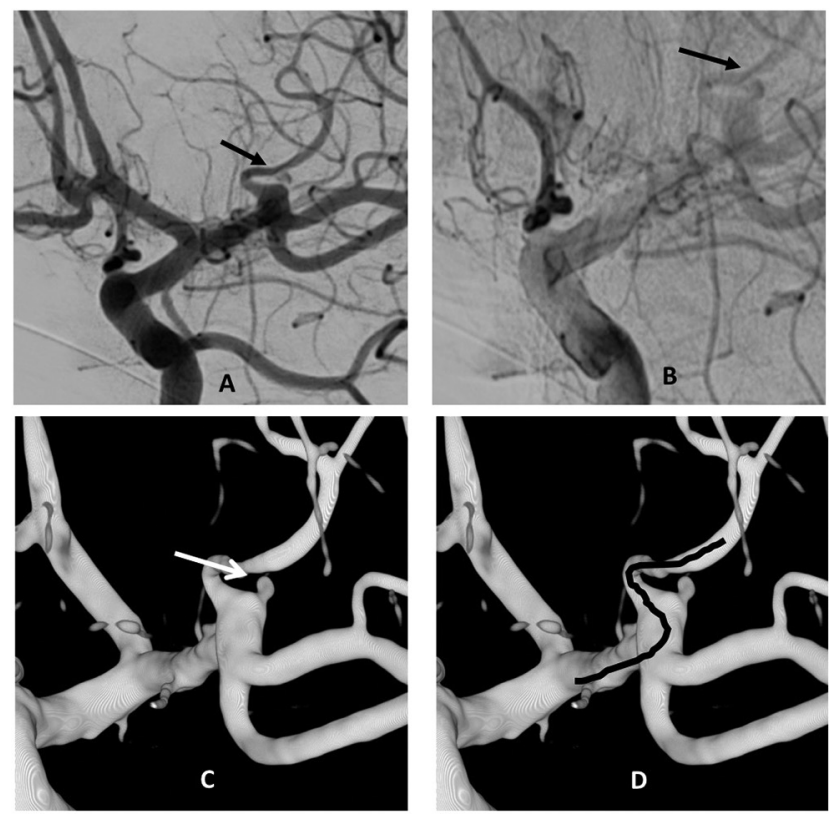

Fig. 1 (A) Diagnostic angiogram showing focal fusiform dilatation at the MCA trifurcation with bleb-like blister aneurysm arising from it (arrow). (B) Delayed phase angiogram showing contrast stasis in blister aneurysm (arrow). (C) Surface-shaded 3D rotational angiogram image showing detailed morphology of the aneurysm in relation to the superior division of the MCA (arrow). (D) Proposed plan of stent deployment with shelfing technique to cover the fusiform base of the aneurysm (black line).
Hyperacute curve was given to the microwire tip (Synchro) to catheterize the superior division of MCA. For aneurysm catheterization, manual shaping of the microcatheter tip was done to adapt to the MCA curves and aneurysm direction, based on 3D rotational angiography. Two curves were given; the proximal curve to adapt to the concave anterior curve of MCA-M1 segment and the distal curve perpendicular to the

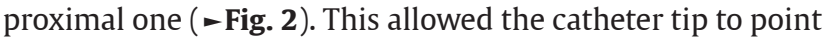
superiorly toward the aneurysm sac and negated the need to place microwire into the sac. Microcatheter position was further adjusted over the first few loops of the coil. The careful consideration was given to coiling microcatheter position and angulation relative to aneurysm for having stable position during coiling along with stent deployment.

Leo Plus Baby (Balt Extrusion) stent $(2.5 \times 25 \mathrm{~mm})$ was deployed partially with shelfing near the neck of the aneurysm. Shelfing technique involves partial deployment of stent till the neck of the aneurysm. Thereafter both microcatheter and stent were loaded to form bulge to cover the base of fusiform dilatation. This allowed an effective covering of the fusiform base with single stent and provided support to coil mass during further coiling. Deployment of the stent in superior division in our case also resulted in remodeling of artery.

With partial deployment of stent, multiple coils were used for the embolization of the blister aneurysm as well as fusiform base of aneurysm. Stent deployment was completed with proximal extent till the left MCA-M1 segment. Post-procedure angiogram showed complete occlusion of the bleb with minimal filling of the fusiform base and good wall apposition of the stent ( $\boldsymbol{- \text { Fig. }}$ 3). Post-extubation, no
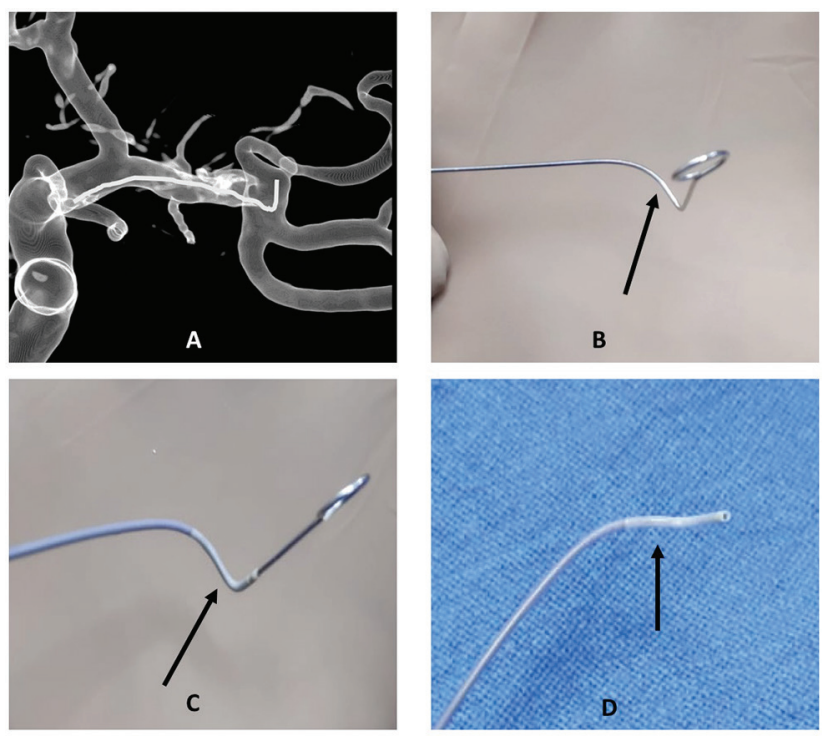

Fig. 2 (A) Three-dimensional (3D) transparent images showing the proximal curve of the MCA and aneurysm direction relative to the parent artery (white line). (B) Shape of the introducer mandrel prior to microcatheter introduction showing the proposed tip curve for microcatheter (arrow). (C) Microcatheter tip with shaping mandrel prior to steaming (arrow). (D) After removal of mandrel and steam shaping, microcatheter tip has adapted to the proposed curvature of proximal MCA as well as aneurysm sac direction for the catheterization (arrow). 

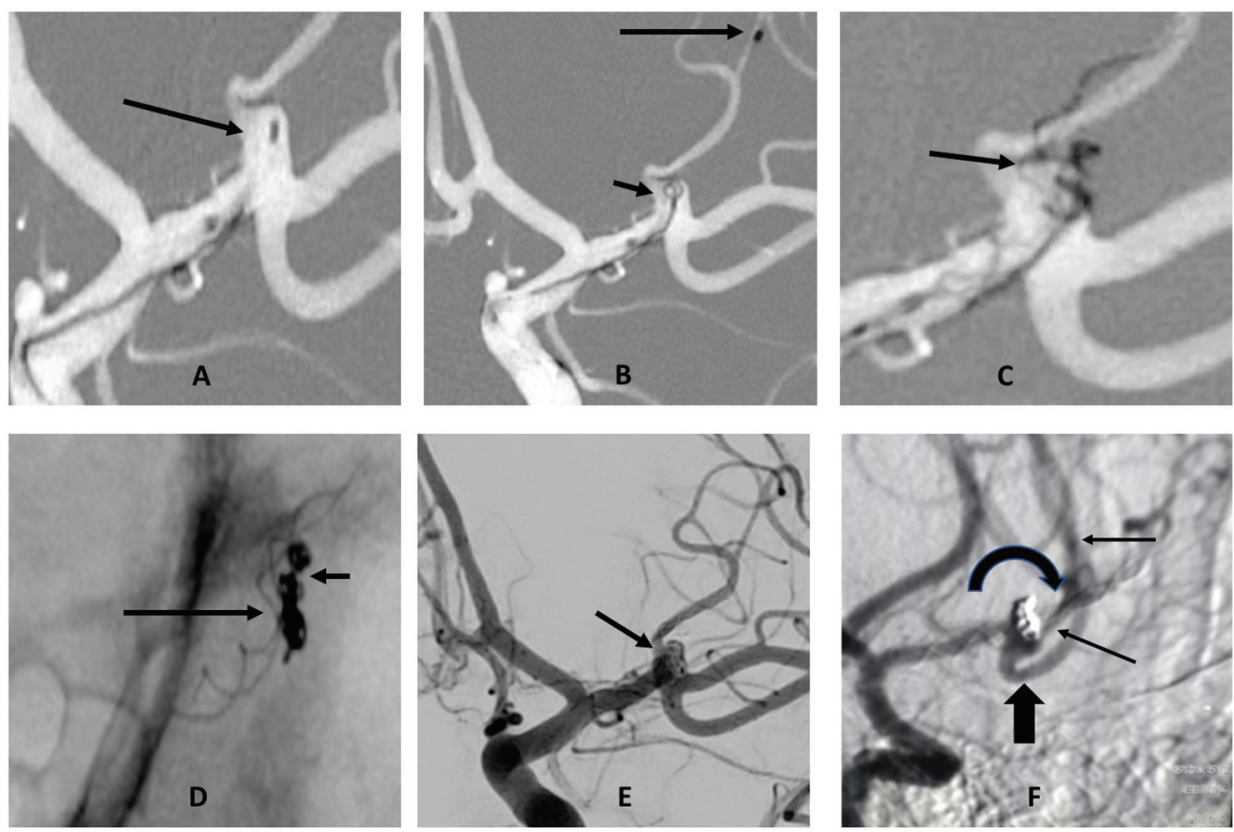

Fig. 3 (A) Roadmap image during the procedure showing coiling microcatheter inside the aneurysm sac (arrow). (B) Roadmap image showing microcatheter for stent deployment in superior division of the MCA (long arrow) and coiling microcatheter with few coil loops inside the aneurysm sac (short arrow). (C) Roadmap image showing the partial deployment of the stent with shelfing near the fusiform base of the aneurysm (arrow). (D) Native image showing the deployed stent with shelfing near the aneurysm base (long arrow) and coil mass inside the aneurysm sac (short arrow). (E) Final angiogram showing complete occlusion of blister with minimal filling of fusiform base (arrow) and preserved flow in all three divisions of the MCA. (F) Six-month follow-up angiogram showing complete aneurysm occlusion along with remodeling of the superior division of the MCA (curved arrow). Preserved flow into middle division (single long arrows) and inferior division (single short thick arrow) of MCA is noted.

neurologic deficits were noted. The patient had gradual recovery over next 2-week period and discharged with GCS of 14 and modified Rankin's scale (mRS) of 1.

Six months follow-up angiogram showed complete occlusion of the aneurysm as well as fusiform base (Raymond-Roy class 1 ) with parent artery reconstruction (superior division of the MCA). The patient had good clinical outcome with mRS of 0 .

\section{Discussion}

Incidence of the blood blister-like aneurysms (BBA) is rare and seen in 0.3 to $1 \%$ of all intracranial aneurysms. ${ }^{2}$ They are known to be associated with young age, female sex, and systemic hypertension. ${ }^{4}$ Etiology is considered to be secondary to hemodynamic stress, underlying atherosclerosis, and possible dissection. On pathology, these are aneurysms with very thin wall covered with fibrin and lacking collagenous tissue layer. ${ }^{3}$ This results in their rapid regrowth and high tendency to rupture if not treated.

Among the locations, supraclinoid ICA is the most common site for blister aneurysm with involvement of ACOM and basilar artery in few case series. MCA is a rarely reported site for the blister aneurysm. Only one case series of three patients treated with both surgical and endovascular method has been reported in the English literature. ${ }^{8}$

Digital subtraction angiography (DSA) with 3D rotational angiography remains gold standard for the diagnosis of blister aneurysms. Lehman et al have reported use of magnetic resonance imaging (MRI) of the vessel wall, which showed T1 hyperintense clot overlying the aneurysm wall. ${ }^{9}$ Smaller size, fragile wall, wide neck, and adjacent parent artery involvement pose significant challenge for the management of this aneurysm. Surgical options are direct clipping, clipping with wrapping of the aneurysm sac, trapping, direct suturing, and if required, extracranial-intracranial bypass. Recent analysis of all surgical technique in blister aneurysm has showed significant intraprocedural rupture and morbidity as well as recurrence. ${ }^{10}$ Modern endovascular devices such as flow diverter and overlapping stents has shown promising results. ${ }^{1,7}$ Flow diverter is the emerging treatment option for the ICA blister aneurysms.

For the management of our case, we considered both surgical and endovascular options. Surgical clipping was considered to have high risk of intraprocedural rupture whereas wrapping has possible risk of re-rupture with subsequent morbidity. Overlapping stents, flow diverter in the superior division, Y-stenting, and simple coiling were considered as endovascular treatment options. Certain morphologic features of the adjacent vessels can have significant impact on the endovascular treatment selection. Our patient had kink in the superior division of the MCA (which was predominantly involved by aneurysm neck). Flow diverter stent can have issues in opening, especially in small tortuous segment vessels. Long-term patency of covered inferior division after flow diverter placement was also questionable. Another option of complex reconstruction using Y-stenting was considered. However, the recent review has showed significantly 
technical difficulties and procedural complications in these complex reconstructions. ${ }^{11}$ Hence Leo Plus Baby assisted coil embolization with shelfing technique was considered suitable option for our patient.

Leo Plus Baby is self-expandable braided stent with closed cell construction. They have a relatively smaller cell size ( $0.9 \mathrm{~mm}$ in Leo Plus Baby) in comparison to laser cut intracranial stents. ${ }^{12}$ Therefore, they have high pore density and low porosity. The cell size changes with the deployment techniques and forces applied along the stent. This property of braided stents is helpful to create a "shelf" to support coil mass using a single stent at the neck of a wide-necked bifurcation aneurysm and therefore excludes the need for $\mathrm{Y} / \mathrm{X}$-stenting. Partial deployment of the stent allowed movement of coiling microcatheter during the procedure. It helped in reconstruction of the branch artery as well as neck of the aneurysm with possible flow diversion effect in long-term leading to aneurysm occlusion. ${ }^{12}$

Shelfing technique with single-braided stent to avoid complex Y-reconstruction of wide neck bifurcation aneurysm is reported with LVIS Jr stent (MicroVention; Terumo). ${ }^{13}$ To our knowledge, this is the first case report of MCA bifurcation blister aneurysm treated with Leo stent monotherapy using shelfing technique.

In conclusion, MCA bifurcation/trifurcation is a rare location of the blister aneurysm. These can be effectively treated with braided stent monotherapy-assisted coil embolization. These microstents can have flow diverter effect secondary to their high pore density. Shelfing technique using these stents achieves favorable parent artery reconstruction with single stent. Our experience also highlights the utility of microstents (size 2-3 mm) in smaller arteries, which may help in parent artery reconstruction.

\section{Source(s) of Support}

None.

\section{Presentation at a Meeting}

None.

\section{Conflicts of Interest}

None.

\section{References}

1 Chinchure SD, Gupta V, Goel G, Gupta A, Jha A. Subarachnoid hemorrhage with blister aneurysms: endovascular management. Neurol India 2014;62(4):393-399

2 Abe M, Tabuchi K, Yokoyama H, Uchino A. Blood blisterlike aneurysms of the internal carotid artery. J Neurosurg 1998;89(3):419-424

3 Ishikawa T, Nakamura N, Houkin K, Nomura M. Pathological consideration of a "blister-like" aneurysm at the superior wall of the internal carotid artery: case report. Neurosurgery 1997;40(2):403-405, discussion 405-406

4 Jha AN, Gupta V. Blister aneurysms. Neurol India 2009;57(1):2-3

5 Andaluz N, Zuccarello M. Blister-like aneurysms of the anterior communicating artery: a retrospective review of diagnosis and treatment in five patients. Neurosurgery 2008;62(4):807-811, discussion 811

6 Rouchaud A, Brinjikji W, Cloft HJ, Kallmes DF. Endovascular treatment of ruptured blister-like aneurysms: a systematic review and meta-analysis with focus on deconstructive versus reconstructive and flow-diverter treatments. AJNR Am J Neuroradiol 2015;36(12):2331-2339

7 Mokin M, Chinea A, Primiani CT, et al. Treatment of blood blister aneurysms of the internal carotid artery with flow diversion. J Neurointerv Surg 2018;10(11):1074-1078

8 Clkla U, Sadighi A, Bauer A, Başkaya MK. fatal ruptured blood blister-like aneurysm of middle cerebral artery associated with Ehlers-Danlos syndrome type VIII (periodontitis type) J Neurol Surg Rep 2014;75(2):e210-e213

9 Lehman VT, Brinjikji W, Mossa-Basha M, et al. Conventional and high-resolution vessel wall MRI of intracranial aneurysms: current concepts and new horizons. J Neurosurg 2018;128(4):969-981

10 Kalani MYS, Zabramski JM, Kim LJ, et al. Long-term follow-up of blister aneurysms of the internal carotid artery. Neurosurgery 2013;73(6):1026-1033, discussion 1033

11 Spiotta AM, Lena J, Chaudry MI, Turner RD, Turk AS. Y-stenting for bifurcation aneurysm coil embolization: what is the risk? Stroke Res Treat 2014;2014:762389

12 Cheung NK, Chiu AH, Cheung A, Wenderoth JD. Long term follow-up of bifurcation aneurysms treated with braided stent assisted coiling and complex T- and Y-stent constructs. J Neurointerv Surg 2018;10(6):560-565

13 Du EHY, Shankar JJS. LVIS Jr 'shelf' technique: an alternative to Y stent-assisted aneurysm coiling. J Neurointerv Surg 2016;8(12):1256-1259 